AIAA 2001-4054

\title{
AN AIRBORNE CONFLICT RESOLUTION APPROACH USING A GENETIC ALGORITHM
}

\author{
Stephane Mondoloni, Ph.D." \\ CSSI Inc. \\ Washington, DC \\ Sheila Conway† \\ NASA Langley Research Center \\ Hampton, VA
}

\begin{abstract}
$\underline{\text { Abstract }}$
An airborne conflict resolution approach is presented that is capable of providing flight plans forecast to be conflict-free with both area and traffic hazards. This approach is capable of meeting constraints on the flight plan such as required times of arrival (RTA) at a fix. The conflict resolution algorithm is based upon a genetic algorithm, and can thus seek conflict-free flight plans meeting broader flight planning objectives such as minimum time, fuel or total cost. The method has been applied to conflicts occurring 6 to 25 minutes in the future in climb, cruise and descent phases of flight. The conflict resolution approach separates the detection, trajectory generation and flight rules function from the resolution algorithm. The method is capable of supporting pilot-constructed resolutions, cooperative and non-cooperative maneuvers, and also providing conflict resolution on trajectories forecast by an onboard FMC.
\end{abstract}

\section{Nomenclature}

\begin{tabular}{|c|c|c|}
\hline$F$ & Fitness function & $\begin{array}{l}\mathrm{t}_{\text {conflict }} \\
\mathrm{t}_{\text {duration }}\end{array}$ \\
\hline$F_{\text {traf }}$ & $\begin{array}{l}\text { Fitness function due to traffic } \\
\text { conflicts }\end{array}$ & \\
\hline $\mathrm{F}_{\text {area }}$ & $\begin{array}{l}\text { Fitness function due to area } \\
\text { conflicts }\end{array}$ & $\tau_{\mathrm{d}}$ \\
\hline $\mathrm{F}_{\text {Pref }}$ & $\begin{array}{l}\text { Fitness function representing user- } \\
\text { preferences }\end{array}$ & $\begin{array}{l}\mathrm{U} \\
\mathrm{x}\end{array}$ \\
\hline $\begin{array}{l}\mathrm{F}_{\text {reduced-l }} \\
\mathrm{h}_{\text {nominal }} \\
\mathrm{h}_{\max }\end{array}$ & $\begin{array}{l}\text { Reduced fitness of } i^{\text {th }} \text { flight plan } \\
\text { Altitude of the nominal trajectory } \\
\text { Maximum altitude of a maneuver }\end{array}$ & \\
\hline
\end{tabular}

\begin{tabular}{|c|c|}
\hline N_group & $\begin{array}{l}\text { Number of waypoints in } \\
\text { maneuver }\end{array}$ \\
\hline n_conflicts & Number of conflicts \\
\hline$P_{\text {add }}^{-}$ & Probability of adding a waypoint \\
\hline$P_{i}$ & $\begin{array}{l}\text { Probability of selection of } i^{\text {th }} \text { flight } \\
\text { plan for perturbation }\end{array}$ \\
\hline$P_{m v}$ & $\begin{array}{l}\text { Probability of perturbing a } \\
\text { waypoint }\end{array}$ \\
\hline$P_{m v_{-} \text {off }}$ & $\begin{array}{l}\text { Probability of perturbing } \\
\text { waypoint off-track }\end{array}$ \\
\hline$P_{\text {sel }}$ & $\begin{array}{l}\text { Probability of selecting a flight } \\
\text { plan for survival }\end{array}$ \\
\hline pop_size & $\begin{array}{l}\text { Number of flight plans in } \\
\text { generation }\end{array}$ \\
\hline $\mathrm{q}$ & Selective pressure \\
\hline$q_{\text {total }}$ & $\begin{array}{l}\text { Minimum distance to leftmost or } \\
\text { rightmost corner }\end{array}$ \\
\hline$r$ & Random variable \\
\hline rank & $\begin{array}{l}\text { Fitness rank of a flight plan in a } \\
\text { generation (e.g., } 1 \text { for best) }\end{array}$ \\
\hline$\sigma$ & Standard deviation \\
\hline$t_{\text {conflict }}$ & Time of start of traffic conflict \\
\hline $\mathrm{t}_{\text {duration }}$ & Duration of traffic conflict \\
\hline$\tau_{C}$ & $\begin{array}{l}\text { Parameter to scale the traffic } \\
\text { conflict start time }\end{array}$ \\
\hline$\tau_{\mathrm{d}}$ & $\begin{array}{l}\text { Parameter to scale the traffic } \\
\text { conflict duration }\end{array}$ \\
\hline $\mathrm{U}$ & Average speed through area hazard \\
\hline$x$ & $\begin{array}{l}\text { Value to be perturbed (spee } \\
\text { altitude) }\end{array}$ \\
\hline
\end{tabular}

${ }^{*}$ Chief Scientist, AIAA Member

†Research Engineer, AIAA Member

Copyright $\odot 2001$ by American Institute of Aeronautics and Astronautics, Inc. No copyright is asserted in the United States under Title 17, U.S. Code. The U.S. Government has a royalty-free license to exercise all rights under the copyright claimed herein for Government Purposes. All other rights are reserved by the copyright owner. 


\section{$\underline{\text { Introduction }}$}

A key part of Distributed Air/Ground (DAG) air traffic management (ATM) research involves the study of separation responsibility between air traffic controllers and flight crew [1]. In order to accomplish this research objective, an airborne decision support tool (DST) to be used in fast-time and human-in-the-loop simulations is being developed. This system is envisaged to provide a plethora of airborne flight planning capabilities to the flight crew such as conflict detection and resolution (CD\&R), flight plan optimization, trial planning and pair-wise separation. This paper focuses on an approach to conflict resolution developed to fit within the framework of the decision support tool under development.

Since the objective of the decision support tool is primarily to conduct research into various operational concepts for DAG-ATM, this DST and all supporting functions must be suitably flexible to investigate variations in the operational concepts as they are imposed. Thus the conflict resolution approach described in this paper was subject to certain requirements as delineated below:

- Conflict-free flight trajectories had to meet flight plan constraints such as required times of arrival (RTA), altitude, and speed constraints.

- Conflict-free flight trajectories had to be "flyable" flight trajectories consistent with those obtained through use of lateral and vertical navigation capabilities on FMS equipped aircraft.

- Conflict resolution should be capable of both cooperative and non-cooperative resolution maneuvers. (Cooperative resolution maneuvers require that all aircraft in a conflict maneuver, whereas non-cooperative maneuvers require that only one aircraft maneuver).

- In the case of non-cooperative maneuvers, the resolution method should be capable of following a set of modifiable flight rules determining which of the conflicted aircraft should maneuver.

- The conflict resolution function must not only resolve aircraft-to-aircraft conflict, but must also resolve encounters with area hazards such as active special use airspace (SUA), inclement weather, terrain, etc.

- The conflict resolution function must be capable of meeting user-specified maneuver preferences such as preferences for lateral maneuvers, or preferences for minimum time maneuvers.

- The conflict resolution function should be capable of being incorporated with a flight plan optimization function.
- The conflict resolution function should be capable of producing a viable solution quickly while still seeking improved solutions.

- The resolution function should resolve conflicts occurring within approximately 20 minutes from the present time.

- Conflict resolution must resolve conflicts occurring during the transition phases of flight. The algorithm must be extensible to all phases of flight.

- The conflict resolution function should allow trial flight plans (flight-crew specified) to be evaluated for conflicts while resolution is being performed on a separate flight plan.

A literature search was undertaken to determine available conflict resolution methods capable of meeting the above objectives. We focused on three types of methods: artificial forces techniques [2-6], the GEARS algorithm (Generic En-route Algorithmic Resolution Service)[8] plus other approaches investigated by Eurocontrol [7,9,10], and a genetic algorithm (GA) approach [11-13]. The ability of each method to meet the above requirements with limited technical risk was considered. Since no existing method could perfectly meet all objectives, some modification to each method would be required. The artificial forces techniques required modifications to incorporate flight plan constraints, area hazards and optimization. The GEARS algorithm required modification to allow cooperative maneuvers, flight plan constraints, and full optimization (optimization not confined to the selection of one flight path from a discrete set). The genetic algorithm required modifications to include cooperative maneuvers and potentially suffered from poor computational performance.

The imposition of flight plan constraints was deemed a more important requirement than the ability to perform cooperative resolutions, thus the genetic algorithm approach was selected with some concepts borrowed from the GEARS algorithm. In addition, cooperative maneuvers can be incorporated by appropriately structuring the "flight rules". One additional advantage of the GA is the ease with which additional constraints and user preferences can be incorporated. The computational performance of the genetic algorithm was not determined to be a problem since prior efforts [11] had obtained suitable performance for the more computationally intensive ground-based problem. 



\section{Description of Approach}

A high-level breakdown of the conflict resolution approach is shown in Figure 1. In a manner similar to the GEARS algorithm, the conflict resolution algorithm is separated from other functions such as conflict detection, flight trajectory generation, and flight rules. Note that the genetic algorithm is contained within the resolution function and operates in a separate thread to the other functions.

A conflict detection function is assumed that maintains current traffic and area hazard information. This conflict detection function determines if a flight trajectory is in conflict with any of the stored area or traffic hazards and returns conflict information. Since multiple conflicts are possible, the conflict detection function returns a list of conflicts for each own-ship trajectory submitted.

A trajectory generation function is required to take an own-ship flight plan with constraints and obtain a flyable trajectory corresponding to that flight plan. The trajectory generation function will include the imposition of turn dynamics, flight plan constraints, and performance constraints. This trajectory generation function may make use of the functionality inherent in an aircraft's flight management computer (FMC). Other information, such as altitude restrictions imposed by the autopilotflight director may be incorporated into this trajectory as well.

A flight rules function is required to accept conflict information and determine whether the own-ship should move according to the rules of flight. The rules function will also determine the maneuver to be attempted by the resolution process along with parameters on that maneuver (e.g. a left vector with maximum deviation of 20 nautical miles). The maneuver to be attempted need not be limited to a single maneuver but may be a combination maneuver such as a vector plus speed control.

The resolution function can accept input data as shown in Figure 1, the processing resulting from the input of each data element is summarized below:
- Upon receipt of user constraints, user preferences or boundary constraints, the data are stored.

- As described in Figure 2, upon receipt of a flight plan with constraints, stored boundary constraints are imposed on the flight plan. This plan is then submitted to the trajectory generation function, the resulting trajectory is submitted to the detection function and tested for conflicts against all area and traffic hazards. If no conflict is detected, a no conflict response is supplied. In the event of a conflict, the conflict information is submitted to the flight rules function. This function then determines if the own-ship must move according to the rules of flight, and what maneuver is required if the ownship must maneuver. If the own-ship must not maneuver, then this output is provided to the flight crew. Otherwise, the resolution function initiates a separate genetic algorithm thread to obtain a conflict-free flight plan subject to user specified preferences and constraints. The separate thread is required to support interrupts and requests for a resolved flight plan while resolution is underway.

- Upon receipt of a trial flight plan with constraints, boundary constraints are imposed on the trial flight plan. The trajectory generation function provides a corresponding trajectory for this flight plan. This trajectory is submitted to a detection function and tested for conflicts against all area and traffic hazards. The results of this detection are supplied to the flight crew.

- While resolutions are being computed, the flightcrew may request the best available resolution. Upon receipt of this request, if a genetic algorithm thread is executing, a message is sent to the genetic algorithm thread requesting the best current resolved flight plan, if available. The GA thread responds with a resolved flight plan, or with a message indicating that no conflict-free flight plan has yet been found. One of the advantages of the GA is the availability of complete solutions prior to convergence. 

Flight Plan ( $w /$ constraints)

\section{User preferences}

User constraints

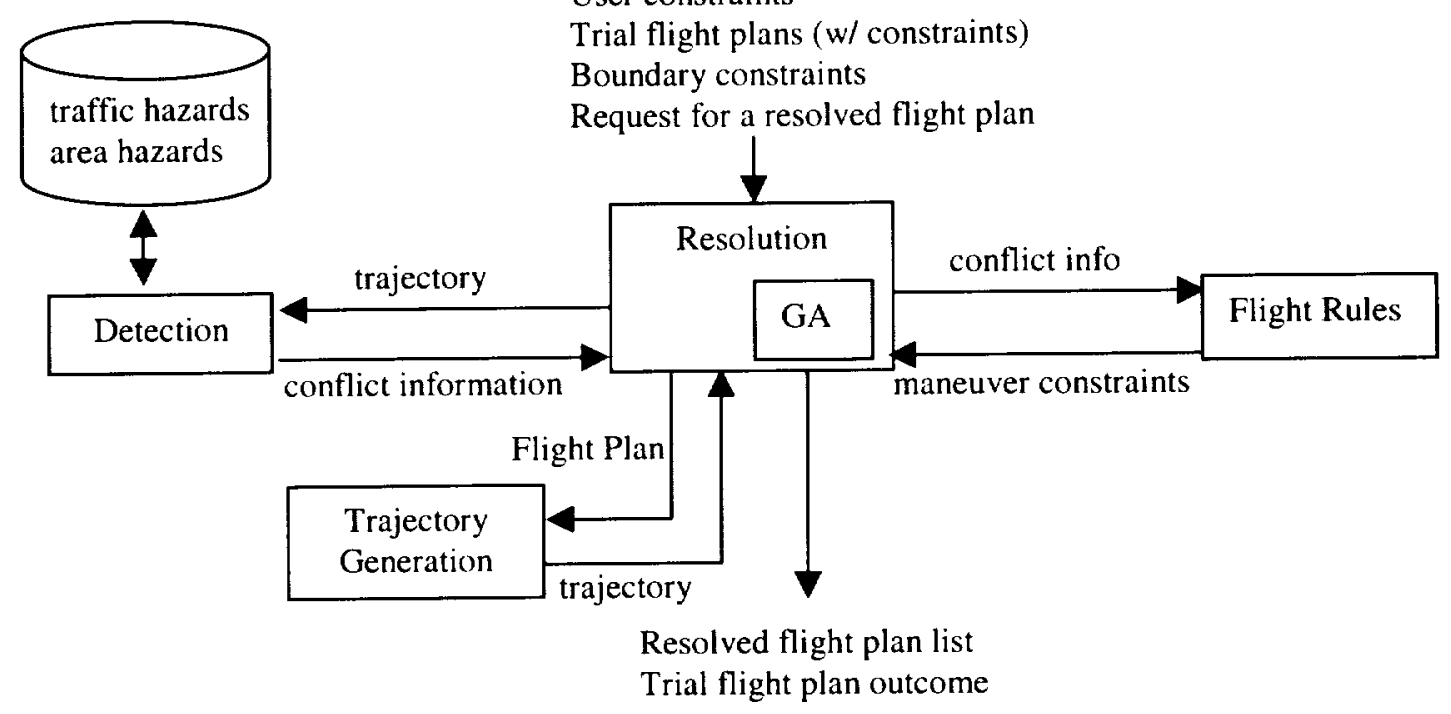

Figure 1. High-level structure of conflict resolution approach.

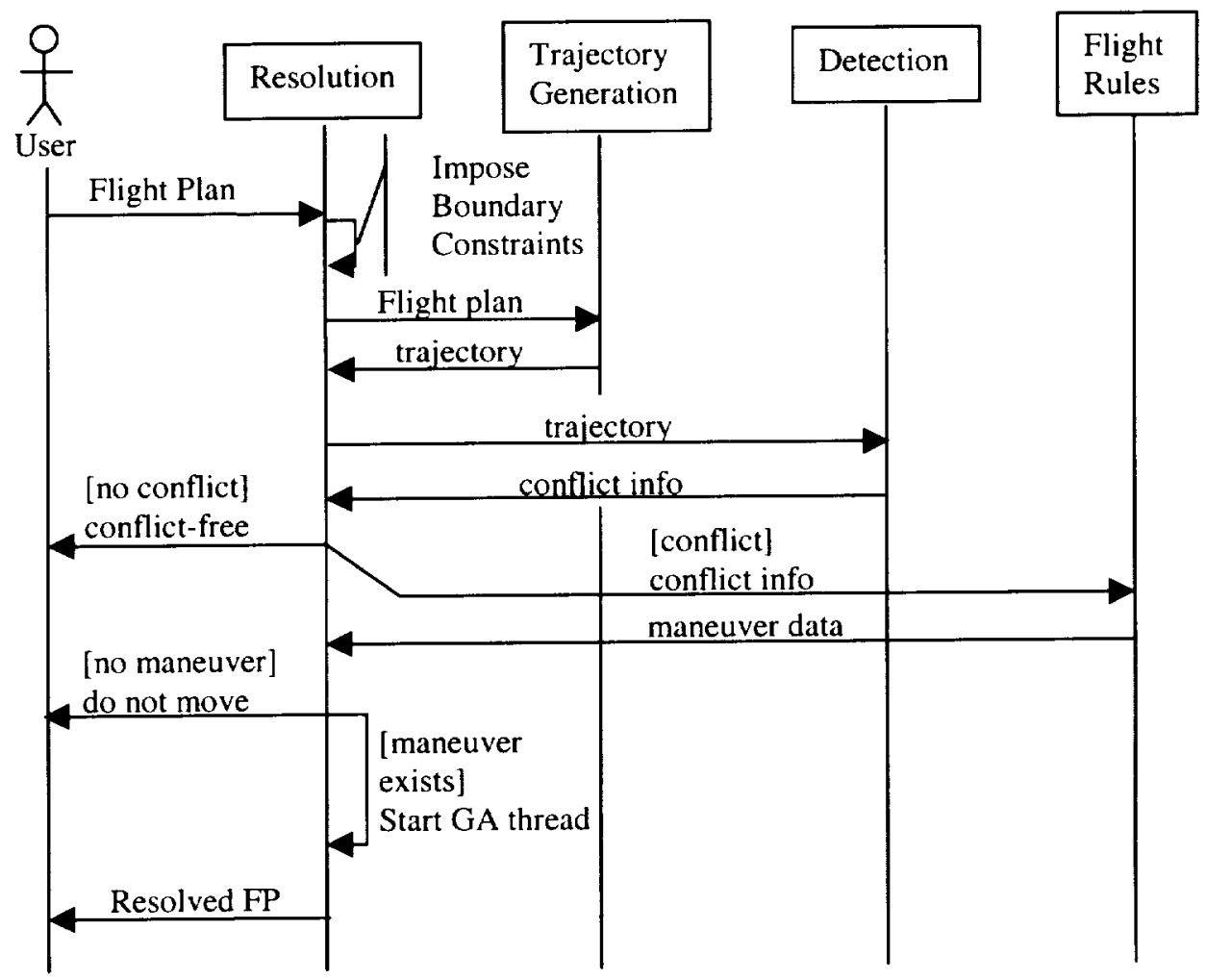

Figure 2. Sequence diagram for receipt of a flight plan with constraints. 


\section{Definitions}

Flight Plan: For the purposes of this report, a flight plan contains a sequence of waypoints with optional altitude, speed and time restrictions at each waypoint. The flight plan also contains global data such as cruise altitude and speed, cost index, and Mach and CAS values for segments during climb and descent. The GA obtains resolutions by modifying the flight plan.

Trajectory: A trajectory is derived from a flight plan. The trajectory describes the forecast fourdimensional path of the flight through a list of time/position data elements. We assume that the flight path may be linearly interpolated between trajectory points. The GA evaluates resolutions by testing the trajectory for conflicts and fitness.

Hazards: Hazards may be area hazards or traffic hazards. Traffic hazards represent intruder aircraft that may conflict with the own-ship. Area hazards represent weather hazards, SUA or terrain.

Conflict Information: A conflict represents the prediction of a loss of separation between own-ship and traffic hazards. The conflict also represents the prediction of penetration of an area hazard by the own-ship trajectory. Conflicts are described through a reference to the conflict hazard (e.g. intruder aircraft identifier), and the starting and ending time of the conflict.

User Preferences: User preferences express which conflict-free flight plan is preferred by the user. Example of preferences include: minimum fuel, minimum time, minimum cost, or minimum additional flight plan constraints.

User Constraints \& Maneuvers: In order to perturb the flight plan, the genetic algorithm uses maneuver information from the flight rules function, or specified by the user. These maneuvers specify the type of maneuver (e.g., vector, altitude change, speed change), the initial direction of maneuver (e.g., left, increase), the maximum magnitudes (e.g., 20 nautical miles), and the starting and ending points of the maneuver on the flight plan.

\section{Summary of Genetic Algorithm}

The genetic algorithm accepts as input a conflicted flight plan and perturbs that flight plan in accordance with a specified maneuver (from the flight rules, or manually input) to produce a conflict-free flight plan meeting the specified constraints and best achieving the user-specified maneuver preferences. The genetic algorithm also uses the trajectory generation and detection functions. Figure 3 illustrates one iteration of the genetic algorithm. The process is summarized below:

a) A sequence of flight plans are perturbed through a series of genetic operators.

b) Boundary constraints (e.g. RTA at a TRACON boundary) are imposed on these perturbed flight plans.

c) Trajectories are obtained for each perturbed flight plan through a trajectory generation function.

d) The conflict detection function is called and then the fitness of each trajectory is evaluated based upon conflict information for conflicted flight plans and user preferences for conflict-free trajectories.

e) The newly evaluated flight plans are combined with the best flight plans from the prior iteration and ranked according to fitness.

f) From these flight plans, copies are randomly selected which will be perturbed in the next iteration. Flights with higher fitness are more likely to be selected.

g) The best flight plans are selected for survival in a probabilistic fashion (higher fitness flight plans have higher probability of selection). In the event of multiple types of maneuvers (e.g. vector or altitude maneuvers) a sharing function is imposed to select a diverse population of flight plans.

Each of these steps is described in more detail below:

\section{Perturbation:}

Perturbations to a flight plan are limited by the maneuver constraints supplied to the genetic algorithm. These maneuver constraints are translated into maneuver envelopes facilitating the perturbation process. The maneuver envelopes are expressed through two flight plans, one representing the maximum allowable deviation in one direction, and the other expressing the maximum allowable deviation in the other direction. These maneuver envelopes can express lateral, speed, time and altitude maneuver constraints. 



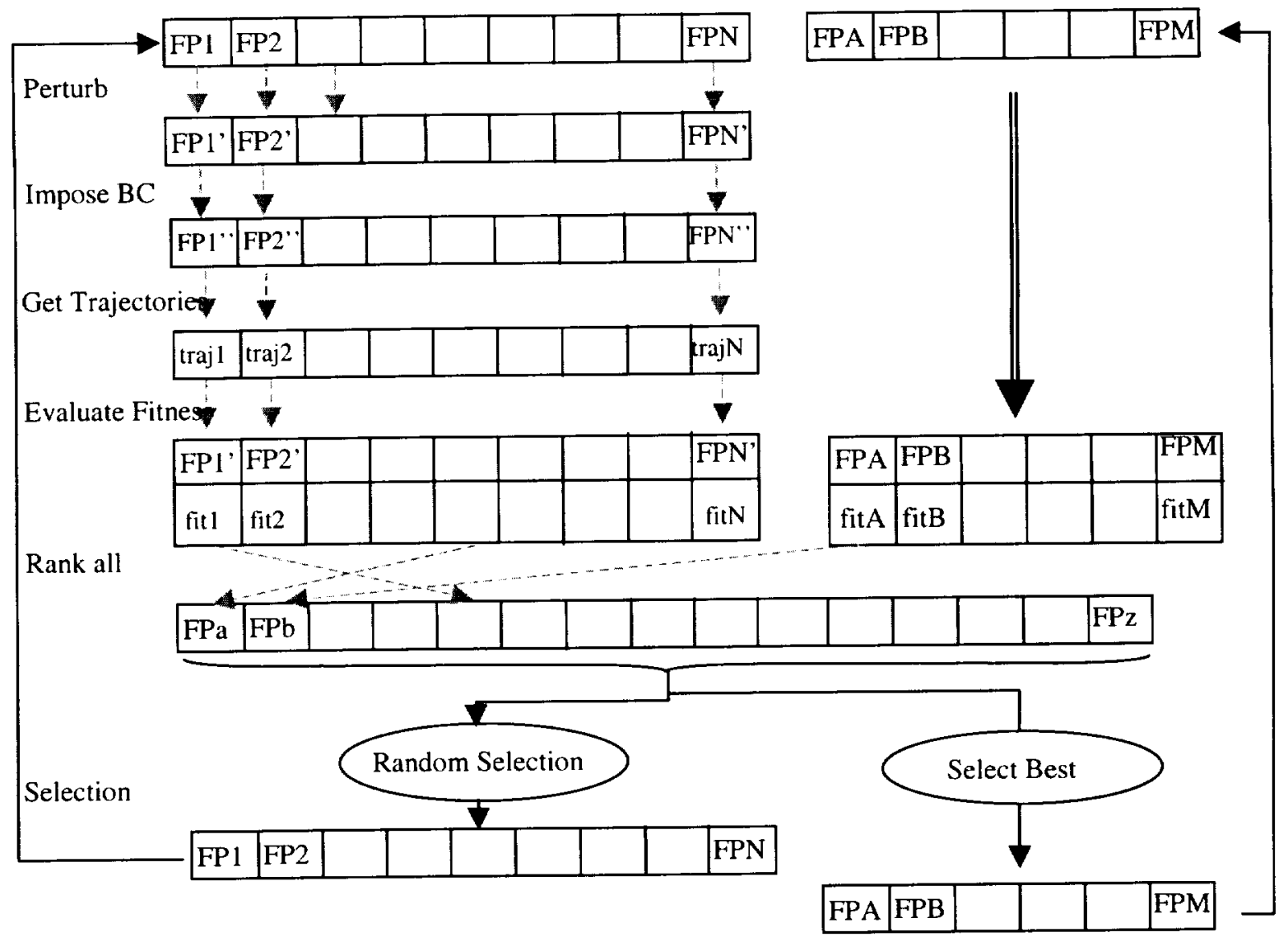

Figure 3. Illustration of one iteration of the Genetic Algorithm.

The process for perturbing a flight plan is sequential beginning with global perturbation. Then, for each point in the maneuver new points are inserted, removed, shortcuts are computed and points are displaced along-track. Finally, points are perturbed across-track and constraints are imposed consistent with the maneuver limits. Since perturbations cannot be executed immediately, a freeze-horizon is required prior to which no perturbations can be introduced.

Each global parameter in a flight plan (e.g., cruise speed, cruise altitude) is perturbed if the value of a random variable selected from a uniform distribution from 0 to 1 , is below a specified probability for that variable. The variable $(\mathbf{x})$ is then perturbed as follows. Note that $r$ is a random variable selected from a normal distribution.

$$
\begin{aligned}
& x^{\prime}= \begin{cases}x+r\left(x_{\max }-x\right) & r>0 \\
x-r\left(x_{\min }-x\right) & r<0\end{cases} \\
& r \sim N(0, \sigma)
\end{aligned}
$$

Waypoints are inserted at a distance (dist) ahead of the current point provided that the next point is beyond the inserted point. The distance is computed using a random number sampled from a uniform distribution as shown below. The term $P_{\text {add }}$ approximates the probability that a point will be added per nautical mile.

$$
\text { dist }=\frac{r \sim(0,1)}{P_{a d d}}
$$

Waypoints are removed from a flight plan if they are not required and a random trial, with specified probability, indicates that the point should be removed.

A shortcut is attempted at each point in a maneuver with specified probability. If a shortcut is to be attempted, all non-required waypoints between the current point and the end of the maneuver are removed.

Each waypoint within the maneuver is then displaced along-track with specified probability. The value of the displacement is obtained from a normal distribution with specified variance. If the point would be displaced beyond the next (or prior) waypoint, the point is placed midway between the old location and the neighbor.

The above process is illustrated in Figure 4. 



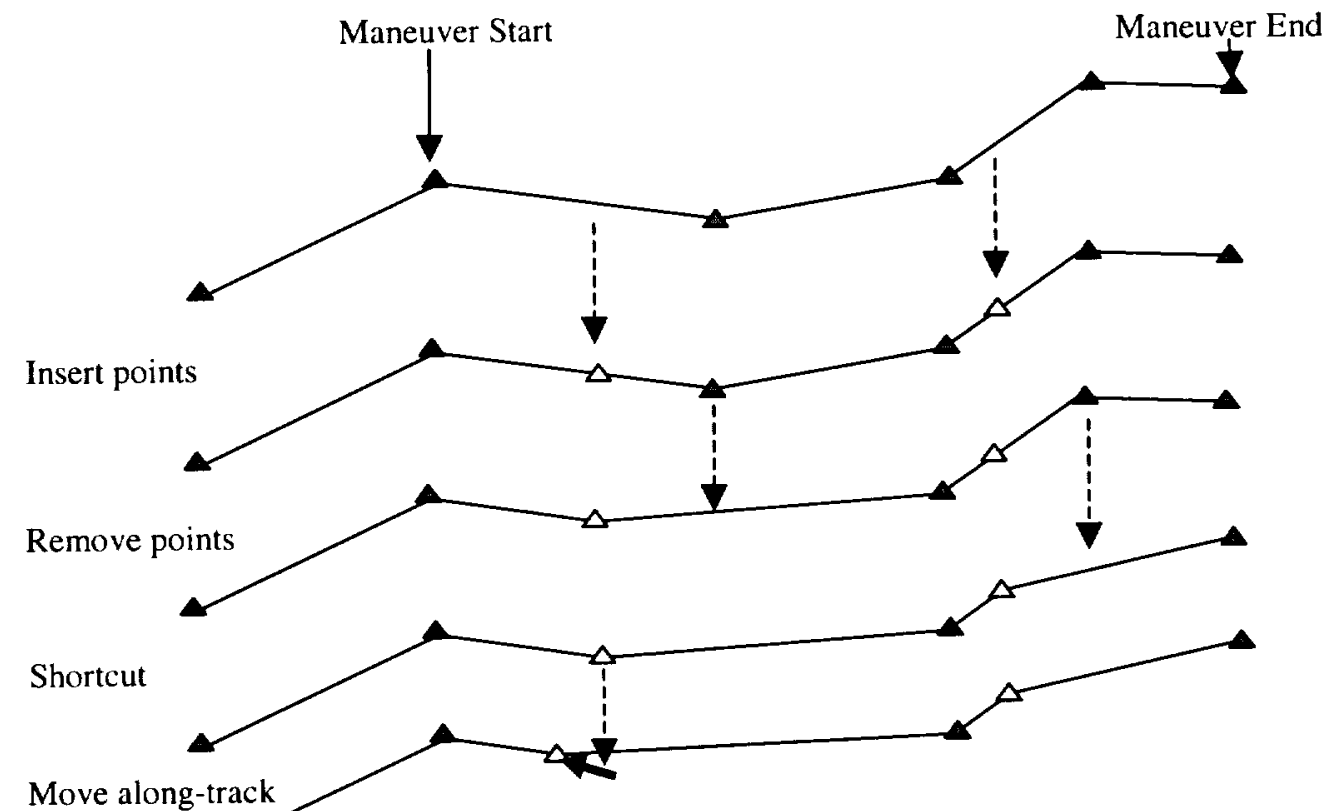

Figure 4. Along-track perturbations

The across-track flight perturbation is illustrated in Figure 5. Points are only displaced off-track if a maneuver allows. If a point is already off-track, and if a random trial indicates, the point is displaced perpendicular to the original flight path according to a random variable selected from a normal distribution with specified variance. If no points are off-track for the desired maneuver, then the probability of a point being selected for displacement is given by:

$$
\left(1-P_{m v}^{N}-\text { group }\right)
$$

Where $\mathrm{N}_{\text {ggroup }}$ is the number of waypoints in the maneuver. The actual waypoint to displace is randomly selected from a uniform distribution from 1 to N_group. The magnitude of the perturbation is limited by the lateral maneuver envelope.

Long wavelength across-track perturbations can be added for generalized lateral maneuvers as shown in Figure 6. The perturbation is introduced at a randomly selected point. The length of the perturbation is also selected from a uniform distribution, but the lateral displacement is selected from a normal distribution with specified variance.

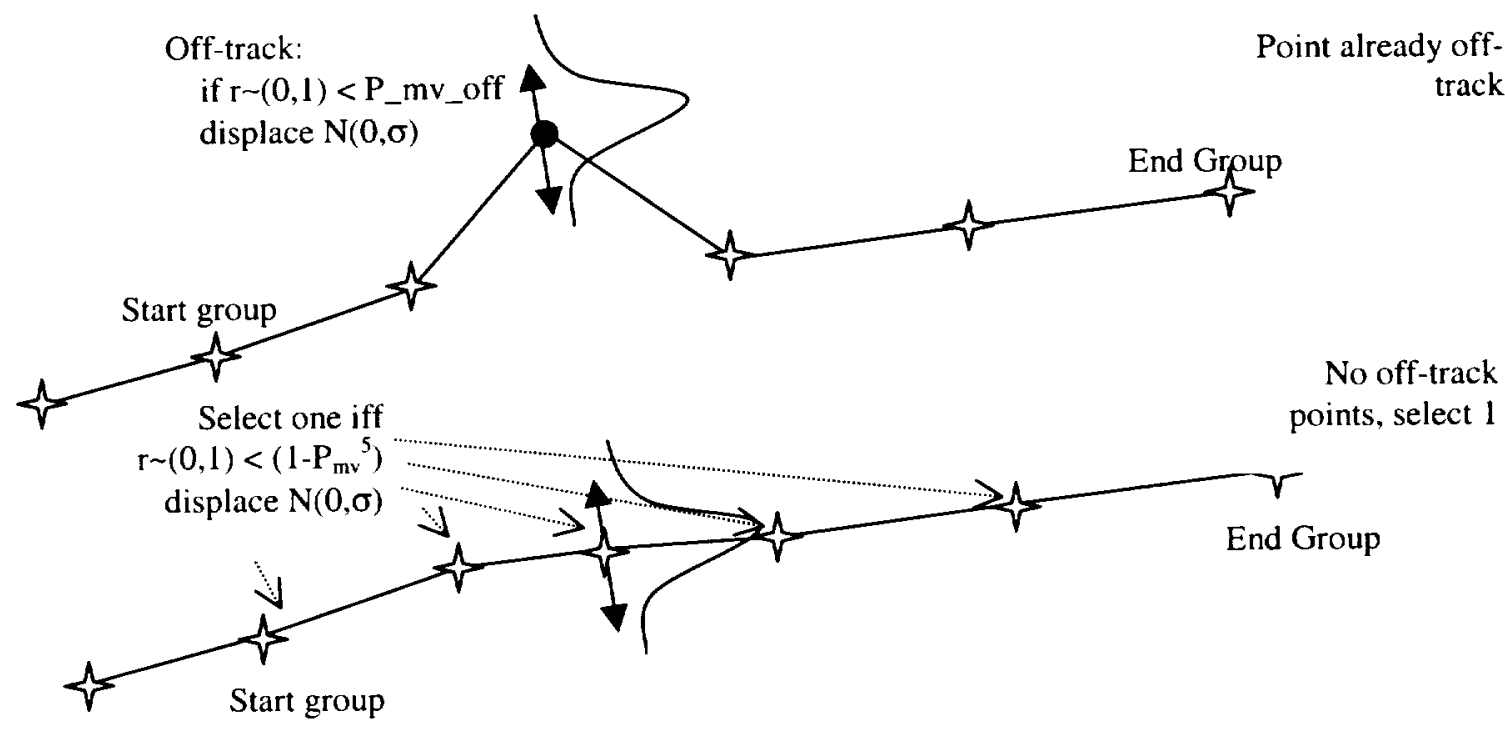

Figure 5. Across-Track perturbation. 



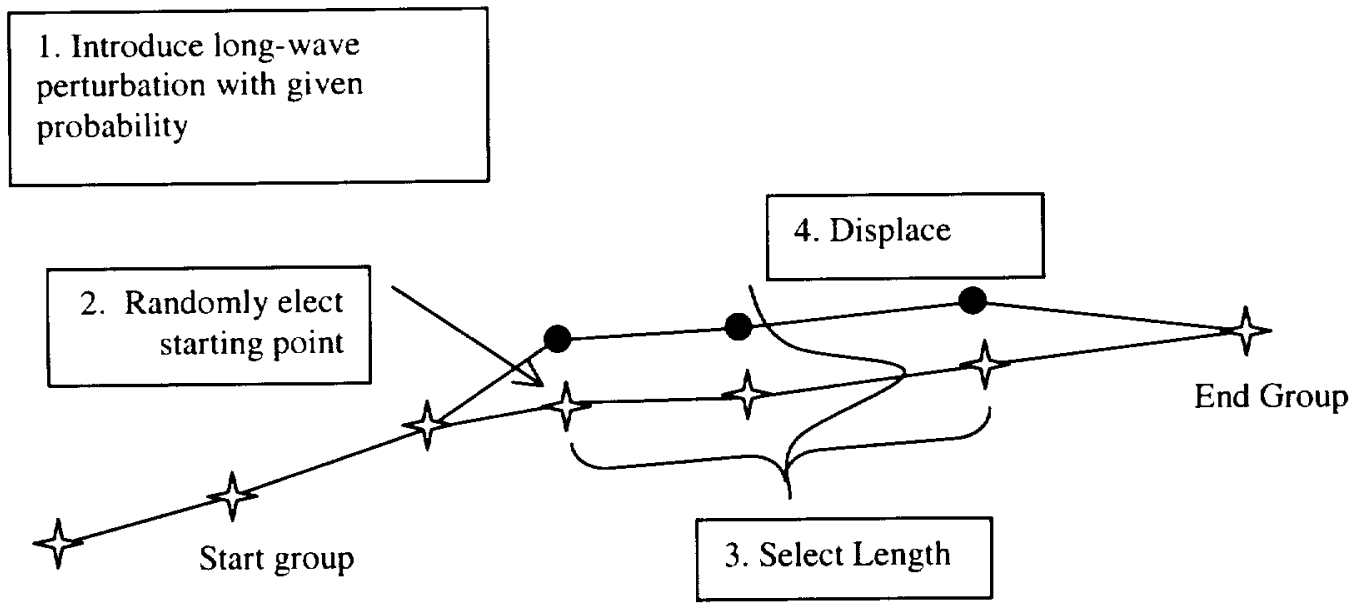

Figure 6. Long-wavelength perturbations

Constraints at waypoints on the flight plan are also imposed through random trials. The constraints can only be imposed at waypoints after the maneuver start, and before the maneuver end. When a point is selected for a constraint (if a uniform random variable is below a specified probability), the constraint is added. For example, an altitude climb would be constrained to a minimum altitude as shown below.

$$
h_{n o \min a l}+N(0, \sigma)\left(h_{\max }-h_{n o \min a l}\right)
$$

\section{Constraints at a Boundary:}

Boundary constraints are imposed on the flight plan in the form of altitude, time or speed restrictions occurring at a boundary. For example, an RTA constraint may be imposed at a TRACON boundary rather than at a specific metering fix. These types of constraints must be imposed subsequent to the perturbation of the flight plan.

\section{Fitness Evaluation:}

The genetic algorithm resolves conflicts by searching for flight plans maximizing a fitness function. In addition to resolving the conflict, the genetic algorithm also seeks the resolved flight plan "best" meeting the user-defined preferences (e.g. minimum time, minimum fuel). The fitness function can be broken down into three components: fitness due to traffic conflicts, fitness due to area conflicts, and fitness due to user preferences. These contribute to the overall fitness as shown below:

$$
F=\left\{\begin{array}{cc}
F_{\text {traf }} F_{\text {area }} & \text { Conflicted } \\
F_{\mathrm{Pr} e f} & \text { Not_conflicted }
\end{array}\right.
$$

Note that the fitness due to preferences will only become effective for flight plans that are conflict free. Conflicted flight plans will have a fitness between 0 and 1 , with one representing a conflict-free flight plan.

Traffic conflicts are obtained through the following multiplicative function.

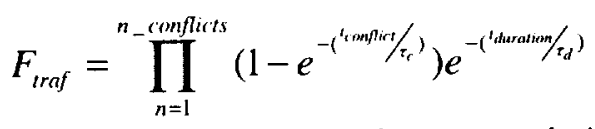

The above fitness function has two desirable properties: the function increases as the conflict duration ( $\left.t_{\text {duration }}\right)$ decreases, and the function increases as the conflict start time $\left(t_{\text {conflict }}\right)$ increases. Thus, maximizing the fitness function will seek flight plans minimizing conflict duration, and also delaying the start of conflict. Two parameters are required to scale the times $\left(\tau_{\mathrm{c}}\right.$ and $\tau_{\mathrm{d}}$ ), these are selected such that maximizing the fitness function seeks to remove conflicts, not just delay them. A contour plot, showing lines of constant traffic fitness versus duration and conflict time, (see Figure 7) illustrates that seeking the maximum fitness function will both remove and delay the conflict.

In order to ensure that conflict duration is an appropriate variable in the fitness function, a collection of conflicts and maneuvers was investigated. Two flights were placed in a variety of conflict situations and a collection of increasing vectors and altitude changes were imposed on one flight. In all cases, the conflict duration was a monotonically decreasing function in one maneuver direction resulting in resolution. 


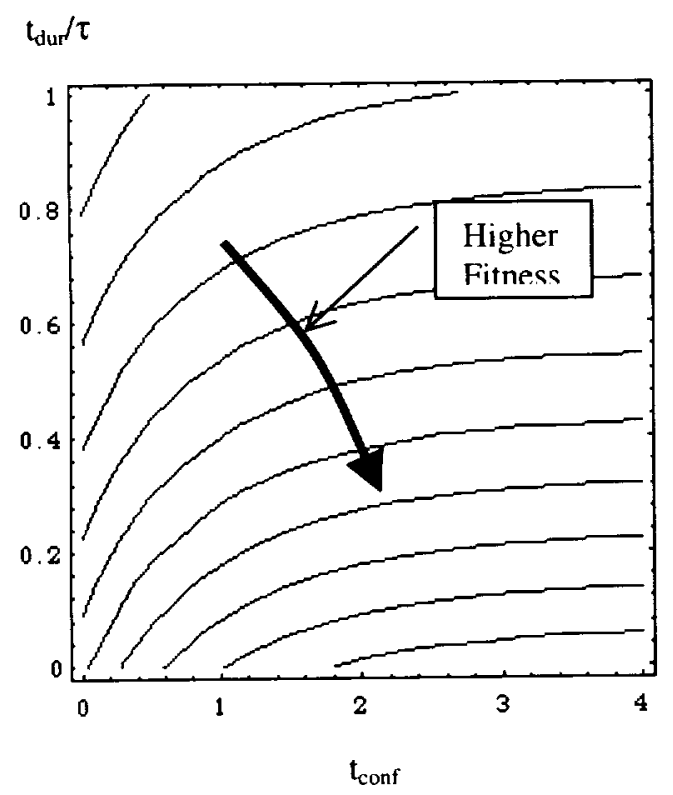

Figure 7. Fitness function as a function of time to conflict and duration of conflict.

While conflict duration provides a simple term for resolution of traffic conflicts, minimizing duration may not resolve conflicts with area hazards. The cost function for area hazards is based upon the timeaveraged perpendicular distance from the SUA entry point to each corner. The furthest corner to the left and to the right of the flight plan is computed. The fitness is based on the minimum distance $\left(\mathrm{q}_{\text {total }}\right)$ of the furthest left or right corner as follows.

$$
F_{\text {area }}=e^{-q_{\text {tomal }} / U}
$$

Where $U$ is the average speed through the area hazard. As the distance to the boundary is reduced to zero, the fitness function is unity, whereas it decreases as the distance to the boundary increases.

The fitness function contribution due to userpreferences is dependent upon the specified userpreference. In all cases, this fitness function will begin at 1 (for the most undesirable flight plan), and grow to a normalized value of 2 for the best flight plan in the generation. Examples of user-preferences include a minimum fuel flight plan, a minimum time flight plan, or a minimum cost flight plan based upon a specified cost index. Note that consistency between the user-preferences and flight plan constraints is required. For instance, specifying a minimum time preference is not consistent with having an RTA at the flight plan end point.

\section{Selection:}

In each generation, flight plans are selected for perturbation according to their "reduced" fitness. The reduction in the fitness is a result of implementing a sharing function. The purpose of the sharing function is to allow breadth in the selection process, thereby preventing "super-fit" individuals from overtaking generations, resulting in premature convergence. In this case, the fitness is reduced by dividing the fitness by the number of flight plans perturbed according to an identical maneuver type. Thus, if $75 \%$ of all flight plans in a generation are perturbed through a vector, and $25 \%$ through an altitude change, the vectored flight plans will be reduced three times as much as the altitude changed flight plans.

The probability of selection of an individual flight plan is based upon the reduced fitness as follows.

$$
P_{i}=\frac{F_{\text {reducet }-i}}{\sum F_{\text {reduced }-i}}
$$

Where the summation is taken over all flights in the generation. Flight plans are selected for perturbation based upon a series of random "wheel-spin" trials according to the above probability for each flight plan.

In addition to selecting flights for perturbation, the best flights from each generation are also selected for survival according to their fitness rank. This second set of flights is not perturbed, but re-enters into the next generation. The probability of selection $\left(\mathrm{P}_{\mathrm{sel}}\right)$ is controlled through a selective pressure (q) as follows.

$$
P_{\text {sel }}=\frac{\sum_{r a n k=1}^{p o p}\left(\frac{1}{q}\right)^{\text {sank-1 }}}{1-\left(\frac{1}{q}\right)^{\text {pop } p_{\text {size }-1}}}(q-1)
$$

A series of random "wheel-spin" trials also determine which flight plans are selected for survival. Flights are only selected once, and once selected, they are removed from the random trial.

\section{$\underline{\text { Results }}$}

The conflict resolution approach described in the preceding section was applied to a collection of traffic conflicts in all following combination of cases ( 243 total). Climb and descent rates were set to 1000 feet per minute. Conflict angles were set to 5,45 and 175 degrees for the parallel-same, crossing and opposite directions respectively. 



\begin{tabular}{|l|l|}
\hline Description & State \\
\hline $\begin{array}{l}\text { Own-ship attitude at } \\
\text { start of conflict }\end{array}$ & climb, cruise, descent \\
\hline $\begin{array}{l}\text { Traffic attitude at start } \\
\text { of conflict }\end{array}$ & climb, cruise, descent \\
\hline $\begin{array}{l}\text { Traffic attitude at end of } \\
\text { conflict }\end{array}$ & climb, cruise, descent \\
\hline $\begin{array}{l}\text { Geometry at start of } \\
\text { conflict }\end{array}$ & $\begin{array}{l}\text { parallel-same, crossing, } \\
\text { parallel opposite }\end{array}$ \\
\hline $\begin{array}{l}\text { Geometry at end of } \\
\text { conflict }\end{array}$ & $\begin{array}{l}\text { parallel-same, crossing, } \\
\text { parallel opposite }\end{array}$ \\
\hline
\end{tabular}

The conflict closest point of approach was selected to occur at 6,18 and 26 minutes from the current point corresponding to the cases of the own-ship in climb, cruise and descent respectively. Initial tests were conducted using rules specifying a vector maneuver for all types of traffic conflicts. All 243 cases resulted in a maneuver specified as conflict-free by the conflict detection function when using a minimum-time user-preference. The mean additional flight time due to the resolution maneuvers was 8 seconds. An example of a resolution maneuver is illustrated in Figure 8 for an opposite direction conflict.

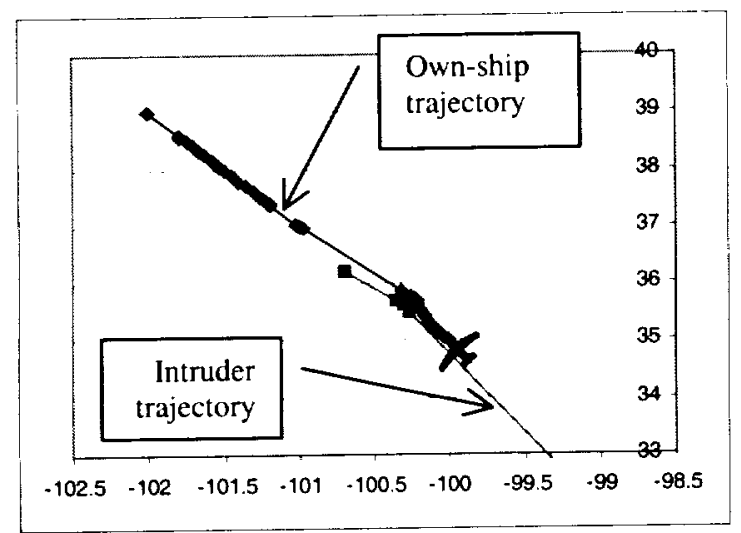

Figure 8. Resolution maneuver to avoid an early opposite direction conflict.

One of the objectives of this conflict resolution approach was to be capable of producing resolutions meeting flight plan constraints such as required times of arrival. We repeated the above cases with an RTA imposed 35 minutes from the starting point. However, a minimum fuel user preference had to be used, as a minimum time user-preference would not be consistent with an RTA. As before, all cases yielded conflict-free maneuvers with a distribution of estimated times of arrival at the RTA point as shown in Figure 9. The sample standard deviation around the mean ETA was 2.3 seconds.

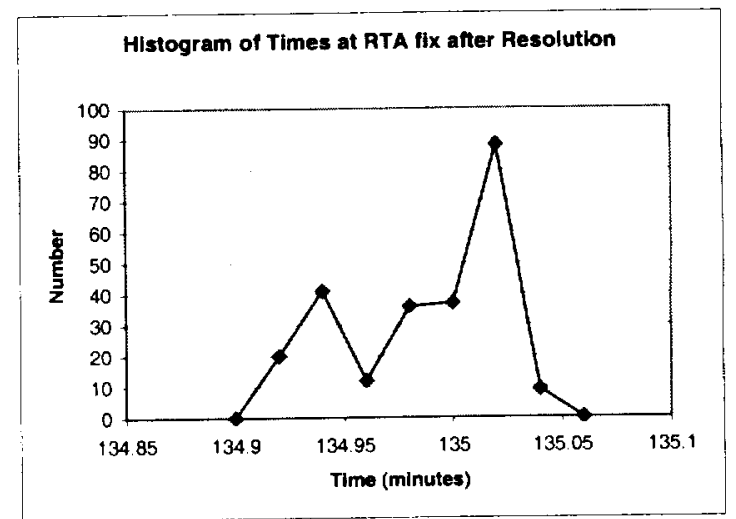

Figure 9. Post-resolution distribution of ETAs at a fix with an RTA set to 135 minutes.

The conflict resolution function was also capable of removing conflicts with area hazards. We attempted lateral area hazard resolution during climb, cruise and descent phases of flight within a range from 6 to 35 minutes from the present time. Both concave and convex area hazards were used. Figure 10 illustrates the resolution trajectories for three simple area hazards. Note that the color of the hazard matches the color of the avoidance trajectory. In all cases, a reasonable direction around the hazard was selected to avoid the hazard.

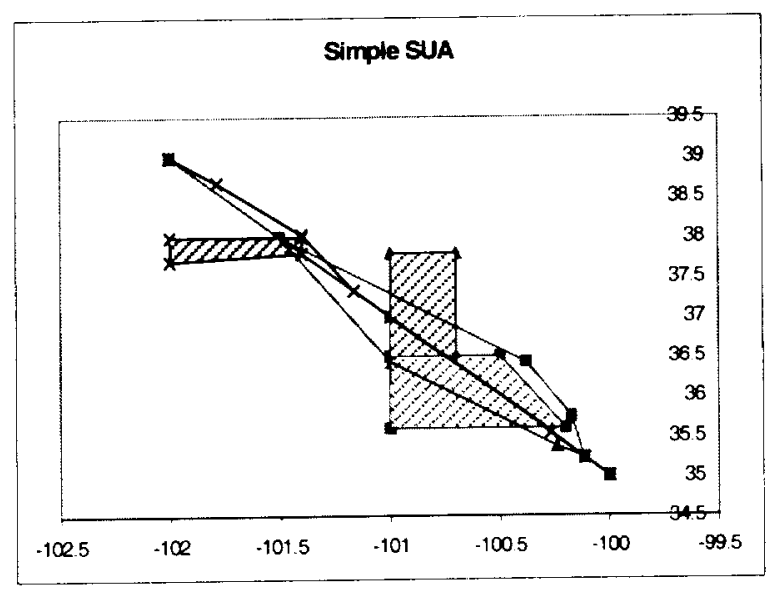

Figure 10. Avoidance maneuvers around area hazards.

One can observe the convergence of the genetic search by looking at the fitness of the "best" flight plan for each generation. Figure 11 illustrates that convergence is achieved through a series of discrete improvements in the flight plan in the case of a minimum time cost function. 



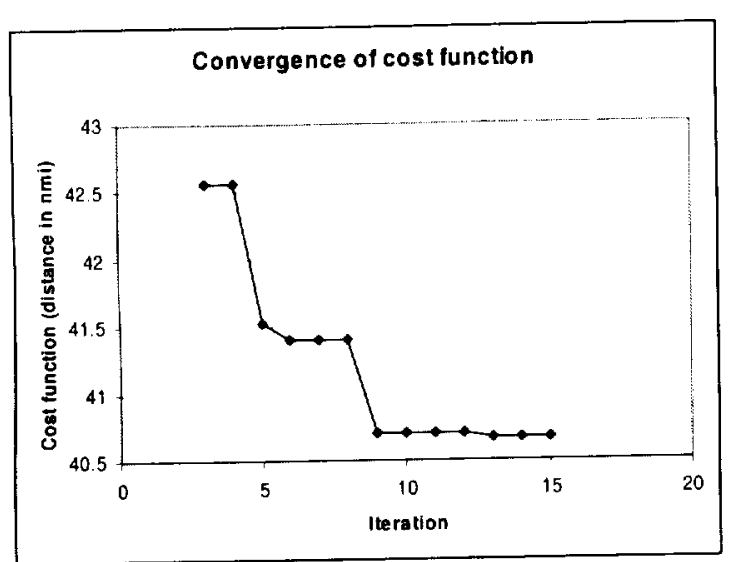

Figure 11. Behavior of best cost function across multiple iterations.

The conflict resolution algorithm was evaluated on a $966 \mathrm{MHz}$ Pentium III. Software was written in $\mathrm{C}++$ and compiled in Microsoft Visual Studio. Performance was not found to be a serious concern as the first resolution was found in a mean of 592 milliseconds (with a standard deviation of $226 \mathrm{msec}$ ). The final resolutions were obtained with a mean of 3.9 seconds and a standard deviation of $129 \mathrm{msec}$.

\section{Conclusion}

An approach to conflict resolution was presented which was demonstrated to be capable of resolving conflicts with both traffic and area hazards. The conflict resolution approach was demonstrated to work in a time horizon of 6 to 26 minutes in multiple phases of flight including transition. The conflict resolution approach was capable of resolving conflicts while still meeting certain flight plan constraints such as required times of arrival at a fix. The conflict resolution approach was demonstrated to work using resolution rules. The algorithm for selecting the maneuver was demonstrated to select resolved trajectories that optimized a user-preferred cost function. As a result, this approach can easily be incorporated with a flight plan optimization function. While not illustrated here, the resolution approach described herein was also demonstrated to be capable of providing manual conflict resolution, and to provide a conflict-free flight plan prior to convergence.

\section{$\underline{\text { References }}$}

[1] Ballin, M.G., et al, “ Airborne Separation Assurance and Traffic Management: Research of Concepts and Technology", AIAA 99-3989.

[2] Eby, Martin S., "A Self-Organizational Approach for Resolving Air Traffic Control Conflicts", Lincoln Laboratory Journal, 1994, Vol. 7 No. 2

[3] Hoekstra, J.M., et al., "Conceptual Design of Free-Flight with Airborne Separation Assurance", AIAA GN\&C Conference, Boston, MA, 1998.

[4] Mackintosh, et al, "Self-Separation from the Air and Ground Perspective", $2^{\text {nd }}$ USA/Europe Air Traffic Management $R \& D$ Seminar, Orlando, Dec. 1998.

[5] Zeghal, K., "A Review of Different Approaches Based on Force Fields for Airborne Conflict Resolution", AIAA GN\&C Conference, Boston, MA, 1998.

[6] Gent, R.N.H.W, Hoekstra, J.M., Ruigrok, R.C.J., "Free-flight with Separation Assurance - A man in the loop simulation study", NLR-TP-98286, May 1998.

[7] Duong, V.N., Zeghal, K., "Conflict Resolution Advisory for Autonomous Airborne Separation in Low Density Airspace"

[8] Irvine, R., "The GEARS Conflict Resolution Algorithm", AIAA 98-4236 AIAA $G N \& C$ Conference, Boston, MA, 1998.

[9] Duong, V.N., Hoffman, E.G., "Conflict Resolution Advisory Service in Autonomous Aircraft Operations", $16^{\text {th }}$ IEEE Digital Avionics System Conference, Irvine, CA, 1997.

[10] Nicolaon, J.P., et al, "An overview of ARC 2000 Version 3 from the Operational Point of View", Executive Summary, EEC Report No. 286, Dec. 1995.

[11] Durand, N., Alliot, J.M., Chansou, O., "An Optimizing Conflict Solver for ATC”, October 1995.

[12] Durand, N., Alliot J.M., Chansou, O.,"Optimal Resolution of En Route Conflicts", Air Traffic Control Quarterly, Vol.3, 1996, pp. 139-161

[13] Gerdes, I., "Construction of Conflict-Free Routes for Aircraft in Case of Free-Routing with Genetic Algorithms", $I^{\text {st }}$ USA/Europe ATM $R \& D$ Seminar, Saclay, 1997.

[14] Michalewicz, "Genetic Algorithms + Data Structures = Evolution Programs", $3^{\text {rd }}$ Edition, Springer, 1996. 
